\title{
Analysis of the efficiency of the Chor-Rivest cryptosystem implementation in a safe-parameter range
}

\author{
L. Hernández Encinas ${ }^{a, 1}$, J. Muñoz Masqué ${ }^{a}$, A. Queiruga Dios ${ }^{b}$ \\ ${ }^{a}$ Department of Information Processing and Coding \\ Applied Physics Institute (IFA), Spanish National Research Council (CSIC) \\ C/ Serrano 144, 28006-Madrid, Spain \\ ${ }^{b}$ Department of Applied Mathematics, ETSII, University of Salamanca \\ Avda. Fernández Ballesteros 2, 37700-Béjar, Salamanca, Spain
}

\begin{abstract}
The Chor-Rivest cryptosystem, based on a high-density knapsack problem on a finite field $\mathbb{F}_{q^{h}}$, was broken by S. Vaudenay for $q \approx 200, h \approx 24$, and $h$ admitting a factor $s$ verifying a certain condition. A new set of parameters $q$ and $h$, which prevent this cryptosystem against Vaudenay's attack, is presented and the computational aspects of its implementation in the Magma computational algebra system are analyzed.
\end{abstract}

Key words: Chor-Rivest cryptosystem, Knapsack problem, Finite fields, Magma software, Public Key Cryptography.

\section{Introduction}

The objective of cryptography is to assure the secrecy and confidentiality of communications and the goal of cryptanalysis is to break the security and privacy of such communications ([12,13]). In particular, in Public Key Cryptography (PKC) each user has two keys: The public key, which is publicly known and it is used by the sender to encrypt a message; and the private key, which is kept in secret by the receiver and it is used by him to decrypt encrypted messages. In general, PKC bases its security on the computational intractability of some Number Theory problems, such as factorization problem, discrete logarithm problem and knapsack problem.

In 1985, Chor proposed a cryptosystem based on the knapsack problem (see $[2,3])$. The last — and the only really efficient - attack to this system has been proposed by Vaudenay ([17]), but only for the parameters originally proposed.

The Chor-Rivest cryptosystem is based on the arithmetic of finite fields and it needs to compute discrete logarithms in order to determine the keys of the

\footnotetext{
${ }^{1}$ Corresponding author. Tel: +34 915618806x458; fax: +34 914117651 .

E-mail addresses: luis@iec.csic.es (L. Hernández Encinas), jaime@iec.csic.es (J. Muñoz Masqué), queirugadios@usal.es (A. Queiruga Dios).
} 
system. The Discrete Logarithm Problem (DLP) can be defined as follows: Given a prime integer $p$, a generator $\alpha$ of the cyclic group $\mathbb{Z}_{p}^{*}$, and an element $\beta \in \mathbb{Z}_{p}^{*}$, the DLP consists in finding an integer $x, 0<x \leq p-1$, such that $\beta=\alpha^{x}$. This problem is considered to be difficult because the best known algorithm for solving it is the number field sieve ([16]) which has a subexponential expected running time:

$$
O\left(e^{\left.\left((64 / 9)^{1 / 3}+o(1)\right)(\ln p)^{1 / 3}(\ln \ln p)^{2 / 3}\right)}\right) .
$$

The security of the cryptosystem depends on the knapsack problem but not on the discrete logarithm problem. In fact, if the DLP becomes tractable, then the Chor-Rivest cryptosystem is easier to implement, but not easier to break.

As other knapsack cryptosystems, Chor-Rivest cryptosystem is not a very popular public key cryptosystem. Some of its drawbacks are that it needs a large time for generating its keys and a big size of public keys.

Nevertheless, nowadays more knapsack-based cryptosystems are being proposed in order to consider new candidates in PKC (see, for example, [18] and the references therein). One of the main reasons argued by the authors to state that their cryptosystem is secure was that it resists the low-density attacks, due to the fact that its density is 1.2 , which is bigger than 0.9408 (see $[1,4,14]$ ). Although the system resists low-density attacks, it was recently broken in [19] by means of a heuristic attack, which permits to recover the private key from the public key. This is an added reason to study the security of some of the most important knapsack proposed, as it is the case of Chor-Rivest cryptosystem.

Moreover, the recommendations for the most popular public key cryptosystems (RSA, ElGamal, etc.) suggest to increase the size of their parameters, due, basically, to the results in quantum computation and in the improvements of the computational time for some number theory problems (see $[9,10])$. In this way, the recommended bitlength of the keys are around 2048-4096 (for example, nowadays there are millions of ID cards in Europe with keys of these sizes).

For these reasons, it is relevant and of interest to study safe alternative cryptosystems, and the Chor-Rivest cryptosystem may be one of them.

In spite of several attacks against Chor-Rivest cryptosystem have been proposed (see $[2,3])$ :

- low-density attacks.

- nothing known (by E. Brickell),

- known $g$ and $r$,

- known $t$ and $r$,

- known $t$ (by O. Goldreich),

- known $\pi$ and $r$ (by A. Odlizko),

none of them is efficient without knowing a part of the private key.

In [17] the Chor-Rivest cryptosystem was broken for the original proposed parameters, i.e., when the cryptosystem is defined over a finite field $\mathbb{F}_{q^{h}}$ with 
$q \approx 200$ and $h \approx 24$. This attack is based on a weakness derived from the fact that the cryptosystem is insecure if the parameter $h$ has a factor $s$ verifying the following condition:

$$
s \geq \sqrt{h+\frac{1}{4}}+\frac{1}{2}
$$

In order to avoid Vaudenay's attack, in [7] a new pair of parameters has been determined for using safely this cryptosystem. In fact, these parameters were computed in a suitable range guaranteeing its security and its computational feasibility; such parameters are $q=409$ and $h=17$. Only a new pair of values was obtained! This is clearly not useful for cryptographic applications.

Here, we present a new set of parameters $q$ and $h$, which prevent Chor-Rivest cryptosystem against Vaudenay's attack. Moreover, the main computational aspects of its implementation in the Magma computational algebra system are analyzed. These results show that there are many (in fact, infinite) values of new parameters for which Vaudenay's attack are unfeasible, which is relevant from a cryptographic point of view. In addition, this new implementation is more efficient than that proposed in [6].

\section{Preliminaries}

A brief description (see $[2,3,7]$ for the details), of the Chor-Rivest cryptosystem is as follows:

1. Choose a prime number $q$ and an integer $h \leq q$ so that the DLP can be efficiently solved in the finite field $\mathbb{F}_{q^{h}}$. This fact is important to generate the keys because this generation needs to compute discrete logarithms in the group $\mathbb{F}_{q^{h}}^{*}$. The reason is that the DLP can be solve in a efficient way by using the Pohlig-Hellman algorithm if the order of the multiplicative group considered, $n=q^{h}-1$, factorizes as a product of small prime factors $([12,15])$.

2. Select a random algebraic element of degree $h$ over $\mathbb{F}_{q^{h}}, t$, and a random irreducible monic polynomial, $f(t)$, of degree $h$, such that $\mathbb{F}_{q^{h}} \approx$ $\mathbb{F}_{q}[t] /(f(t))$.

3. Choose a random generator, $g$, of the group $\mathbb{F}_{q^{h}}$. Such generator can be chosen at random in $\mathbb{F}_{q^{h}}^{*}$ until it verifies $g^{\left(q^{h}-1\right) / l} \neq 1$ for all prime divisors, $l$, of $q^{h}-1$.

4. Compute the discrete logarithms $\log _{g}\left(t+\alpha_{i}\right)=a_{i}, \forall \alpha_{i} \in \mathbb{F}_{q}$.

5. Generate a random permutation of $q$ elements, $\pi:\{0,1, \ldots, q-1\} \rightarrow$ $\{0,1, \ldots, q-1\}$ and compute $b_{i}=a_{\pi(i)}$.

6. Add a random noise, $0 \leq r \leq q^{h}-2$, to obtain the elements of the knapsack: $c_{i} \equiv\left(b_{i}+r\right)\left(\bmod q^{h}-1\right), 0 \leq i \leq q-1$, which are the public key.

7. The private key is formed by the values $(t, g, \pi, r)$. 
The messages to be encrypted, $M$, are binary vectors of length $q$ and weight $h([5])$, i.e.,

$$
M=\left(m_{0}, m_{1}, \ldots, m_{q-1}\right), \quad m_{i} \in\{0,1\}, \quad \sum_{i=0}^{q-1} m_{i}=h .
$$

The ciphertext corresponding to a message $M$ is:

$$
E=\sum_{i=0}^{q-1} m_{i} c_{i} \quad\left(\bmod q^{h}-1\right) .
$$

For decryption, $M$ is obtained from $E$ as follows:

i. Compute $s \equiv E-h r\left(\bmod q^{h}-1\right)$.

ii. Obtain the polynomial of degree $h-1$, given by $Q(t)=g^{s}(\bmod f(t))$.

iii. Determine the $h$ roots, $\alpha_{\pi(i)}$, of $f(t)+Q(t)$ in $\mathbb{F}_{q}$, such that,

$$
f(t)+Q(t)=\prod_{i \in I}\left(t+\alpha_{\pi(i)}\right) .
$$

iv. Apply $\pi^{-1}$ to the roots $\alpha_{\pi(i)}$ for recovering the coordinates of the original message, $M$, having the bit 1 .

\section{New and safer parameters}

The first efficient attack against the Chor-Rivest cryptosystem for the original proposed parameters in [2,3] (i.e., $q \cong 200, h \cong 24$ ), is developed in [17], assuming, in addition, $h$ has a factor verifying the condition (1).

After that, some new parameters have been found, say $q=409, h=17$, keeping the safety of the cryptosystem and some important properties, like density, size of the public knapsack, etc. (see [7] for more information). Nevertheless, this pair of parameters, is too restrictive in order to develop practical implementations. For this reason, in the present study the restrictions considered in [7] have been relaxed in order to determine new pairs of the parameters $(q, h)$ and thus to obtain a larger set of parameters for implementations of the cryptosystem, but keeping the same level of safety and avoiding Vaudenay's attack.

In order to avoid Vaudenay's attack we first need to determine those parameters $h$ for which no divisor $s$ verifies the condition (1). We obtain

Proposition 1. If an integer $h$ has no factor s verifying the inequality (1) then $h$ is either a prime number or the square of a prime number.

Proof. Assume $h$ is not prime and let $k$ be the least prime divisor of $h$. Hence, $h=s \cdot k, s \in \mathbb{Z}, s \geq k$. If $s=k$, the result follows. If $s>k$, then the inequality $s<\sqrt{h+\frac{1}{4}}+\frac{1}{2}$ implies $\left(s-\frac{1}{2}\right)^{2}<h+\frac{1}{4}$, and simplifying $s(s-1)<h=s \cdot k$. Hence, $s-1<k$, thus leading us to $k<s<k+1$, which is not possible.

The conditions for obtaining the new range of parameters are the following: 
(a) $2 \leq h \leq q, q$ being a prime number and $h$ being either a prime number or the square of a prime number.

(b) $10^{35}<n=q^{h}-1$, that is, the number of digits of $n$ is $t(q, h) \geq 36$.

(c) The bitlength of the public key for $(q, h)$ satisfies $l(q, h)<150000$.

(d) The density, $d(q, h)$, for the knapsack is much bigger than 1 , to avoid all known attacks to low-density knapsack cryptosystems.

(e) The smoothness of $n, u(n)$, i.e., the greatest prime factor of $n$ has at most 18 decimal digits: $u(n) \leq 18$.

These conditions are justified taking the following facts into account:

- First, Vaudenay's attack is not applicable if condition (a) is assumed. In fact, as $q$ is a prime number and $h$ is prime or the square of a prime number, $h$ has no factors and the condition (1) has no sense.

- Condition (b) is less restrictive than that considered in [6], but the cryptosystem is still safe because no attack is known under the assumption of conditions (a) and (b).

- Condition (c) has been considered because of Lenstra's conjecture ([11]), which states that knowing the whole public knapsack could be a weakness for the system. Hence, the idea is to publish only a half (or less) of the public key. Note that the cryptosystem still works in this case.

Condition (d) remains unchanged as no outstanding improvements have been published in relation to the attacks against the value of the density of a knapsack. In fact, the improvement published in [8] considers that is possible to break a system if its density is $<1$. But this bound cannot be applied to the results obtained below (see Table 1).

- Finally, condition (e) is considered under the assumption that the number of bit operations per day of a standard PC is around $10^{14}$. This value for the smoothness of $n, u(n) \leq 18$, has been obtained carrying out similar computations to those in [6].

In Table 1 the 44 pairs $(q, h)$ verifying the conditions (a)-(e) are shown.

Table 1: Pairs of parameters $(q, h)$ verifying the conditions (a)-(e).

\begin{tabular}{|c|c|c|c|c|c|}
\hline$h$ & $q$ & $t(q, h)$ & $l(q, h)$ & $d(q, h)$ & $u(n)$ \\
\hline \hline 13 & 547 & 36 & 64546 & 4.626 & 14 \\
\hline 13 & 571 & 36 & 67949 & 4.796 & 13 \\
\hline 13 & 577 & 36 & 68663 & 4.838 & 14 \\
\hline 13 & 599 & 37 & 71281 & 4.994 & 13 \\
\hline 13 & 601 & 37 & 72120 & 5.008 & 11 \\
\hline 13 & 613 & 37 & 73560 & 5.092 & 15 \\
\hline 13 & 631 & 37 & 75720 & 5.218 & 11 \\
\hline 13 & 641 & 37 & 77561 & 5.288 & 17 \\
\hline
\end{tabular}


Table 1: Pairs of parameters $(q, h)$ verifying the conditions (a)-(e).

\begin{tabular}{|c|c|c|c|c|c|}
\hline$h$ & $q$ & $t(q, h)$ & $l(q, h)$ & $d(q, h)$ & $u(n)$ \\
\hline \hline 13 & 659 & 37 & 79739 & 5.413 & 16 \\
\hline 13 & 683 & 37 & 83326 & 5.579 & 14 \\
\hline 13 & 719 & 38 & 88437 & 5.828 & 15 \\
\hline 13 & 757 & 38 & 93868 & 6.088 & 16 \\
\hline 13 & 787 & 38 & 98375 & 6.292 & 15 \\
\hline 13 & 797 & 38 & 99625 & 6.360 & 13 \\
\hline 13 & 839 & 39 & 105714 & 6.644 & 11 \\
\hline 13 & 877 & 39 & 111379 & 6.900 & 16 \\
\hline 13 & 887 & 39 & 112649 & 6.967 & 16 \\
\hline 13 & 941 & 39 & 120448 & 7.327 & 14 \\
\hline 13 & 953 & 39 & 121984 & 7.407 & 16 \\
\hline 13 & 967 & 39 & 123776 & 7.500 & 14 \\
\hline 13 & 977 & 39 & 126033 & 7.566 & 16 \\
\hline 13 & 1093 & 40 & 143183 & 8.329 & 13 \\
\hline 13 & 1123 & 40 & 147113 & 8.524 & 16 \\
\hline 17 & 127 & 36 & 14986 & 1.068 & 15 \\
\hline 17 & 131 & 36 & 15589 & 1.095 & 15 \\
\hline 17 & 167 & 38 & 20875 & 1.330 & 14 \\
\hline 17 & 193 & 39 & 24897 & 1.495 & 17 \\
\hline 17 & 233 & 41 & 30989 & 1.742 & 15 \\
\hline 17 & 263 & 42 & 35768 & 1.924 & 15 \\
\hline 17 & 277 & 42 & 37949 & 2.008 & 15 \\
\hline 17 & 317 & 43 & 44697 & 2.244 & 17 \\
\hline 17 & 331 & 43 & 47002 & 2.326 & 17 \\
\hline 17 & 409 & 45 & 60123 & 2.773 & 9 \\
\hline 17 & 433 & 45 & 64084 & 2.908 & 18 \\
\hline 17 & 587 & 48 & 91572 & 3.754 & 14 \\
\hline 17 & 643 & 48 & 101594 & 4.054 & 17 \\
\hline 17 & 661 & 48 & 105099 & 4.150 & 16 \\
\hline 23 & 173 & 52 & 29410 & 1.011 & 18 \\
\hline 23 & 191 & 53 & 33234 & 1.095 & 18 \\
\hline 23 & 199 & 53 & 34825 & 1.132 & 15 \\
\hline 23 & 283 & 57 & 52921 & 1.510 & 16 \\
\hline 23 & 563 & 64 & 118230 & 2.679 & 14 \\
\hline 25 & 601 & 70 & 138230 & 2.604 & 17 \\
\hline 25 & 613 & 70 & 141603 & 2.648 & 16 \\
\hline
\end{tabular}

Table 2 shows the pairs of parameters for which the $q$ discrete logarithms can be computed over in one day, approximately. This computation has been made by using the Magma implementation presented in $\S 4$, with a Pentium D-930 3.00 GHz, 2.00 GB RAM. In this Table, the column Total Time contains the number 
of seconds of CPU time needed for the calculation of the $q$ logarithms; while the column Average Time is filled with the average time, in seconds, required to calculate each of the $q$ logarithms.

Table 2: Pairs $(q, h)$ for which $\log _{g}(t+i), 0 \leq i \leq q-1$, can be computed in 1 day, approximately.

\begin{tabular}{|c|c|c|c|}
\hline$h$ & $q$ & Total Time & Average Time \\
\hline \hline 17 & 409 & 198.109 & 0.484 \\
\hline 13 & 601 & 3454.592 & 5.748 \\
\hline 13 & 631 & 7308.658 & 11.583 \\
\hline 13 & 839 & 14719.431 & 17.544 \\
\hline 13 & 599 & 32918.392 & 54.956 \\
\hline 13 & 797 & 77935.191 & 97.786 \\
\hline 13 & 571 & 80912.053 & 141.702 \\
\hline 13 & 577 & 88155.129 & 152.782 \\
\hline 17 & 167 & 98539.430 & 590.056 \\
\hline
\end{tabular}

\section{Implementation with Magma software}

Magma is a Computer Algebra System designed to solve problems in algebra, number theory, geometry and combinatorics that may involve sophisticated mathematics and which are computationally hard. Magma provides a mathematically rigorous environment which emphasizes structural computation. The kernel of Magma contains implementations of many of the important concrete classes of structure in five fundamental branches of algebra, namely group theory, ring theory, field theory, module theory and the theory of algebras. That is why we choose Magma, a more appropriate software than that used in [6]. Maple allows to develop mathematics with a more accessible and easy to use software, but Magma is a large, well-supported software package designed to solve computationally hard problems in algebra.

The implementation of Chor-Rivest cryptosystem with Magma software is composed by several procedures. The main procedures are related to the generation of the keys, and to encrypt and decrypt messages. We will present these procedures with detail. Moreover, there are other procedures necessary to transform the messages in a suitable way (see $[2,3]$ ), to determine the generator of the multiplicative group, and to generate the permutation; but they are not an essential part of the cryptosystem implementation.

The public and private keys are generated by means of the procedure keys in the following way. Once they are obtained, the public and the private keys can be saved in two files for their later use.

First of all, the finite field of $q$ elements, $\mathbb{F}_{q}$, is defined, and then an extension of it is considered. After defining the finite field and its extension, it is necessary to compute some discrete logarithms. 


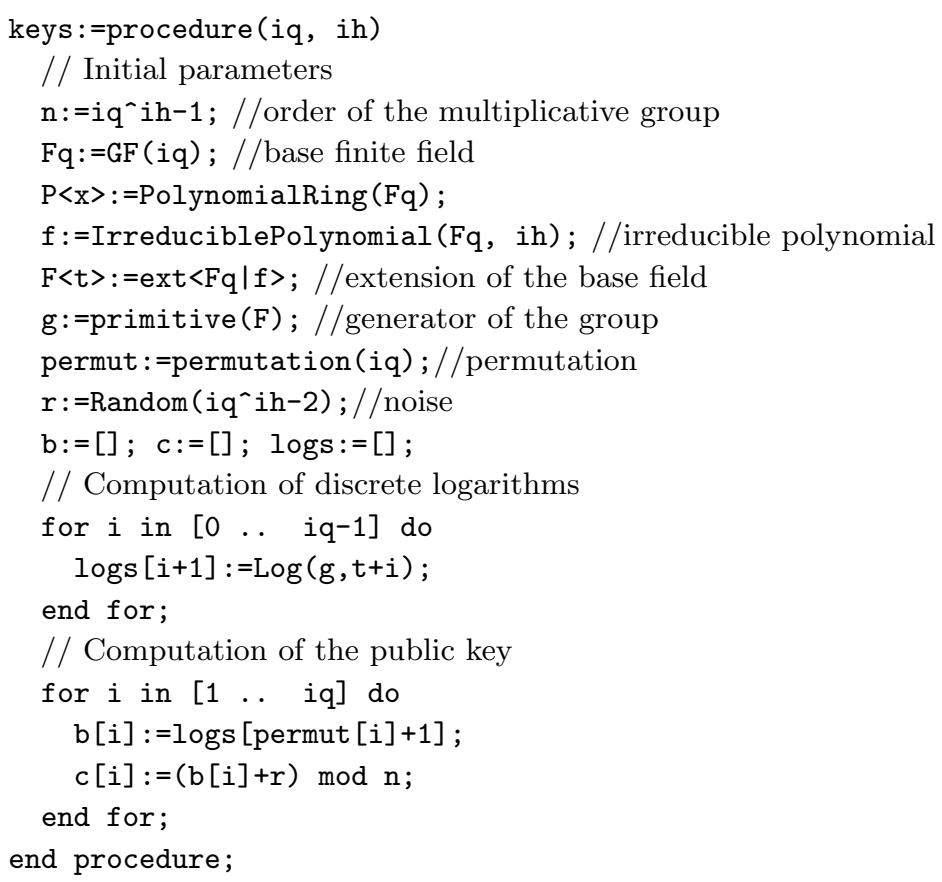

The procedure for encrypting a message is as follows. First of all, the sender considers the text of his message, $M$, computes the length and the number of blocks of the message, transforms and divides the message into blocks of the same length, and transforms it in blocks of length $q$ and weight $h$ (see the corresponding algorithm in [2, 3]). Later, the sender encrypts the message and saves it in a file.

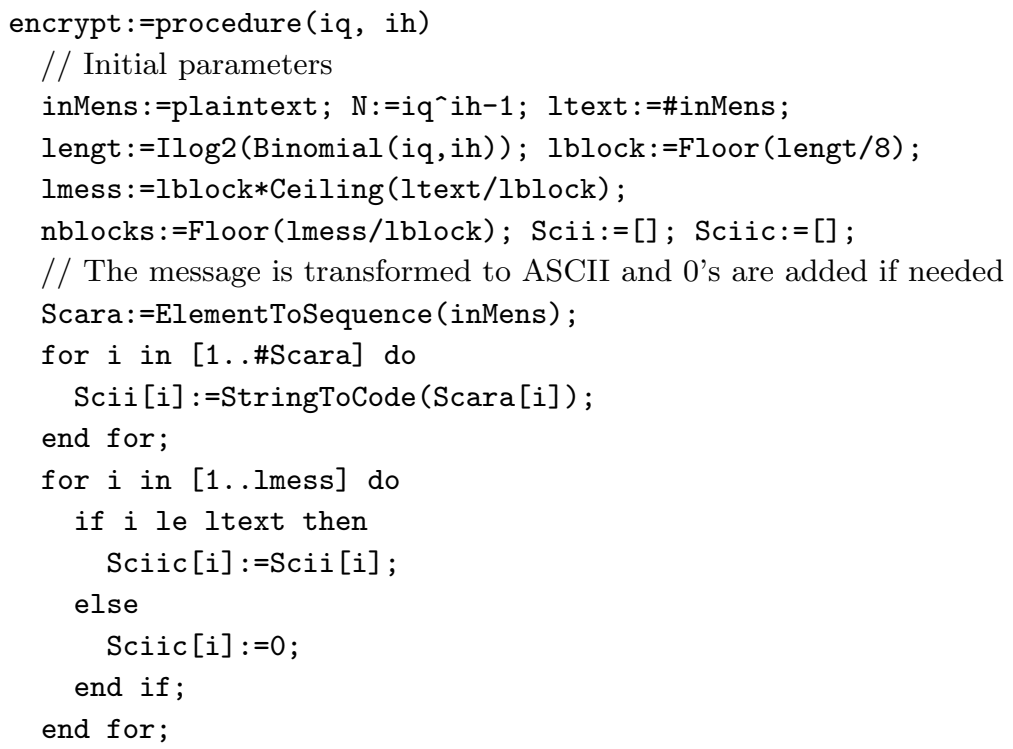




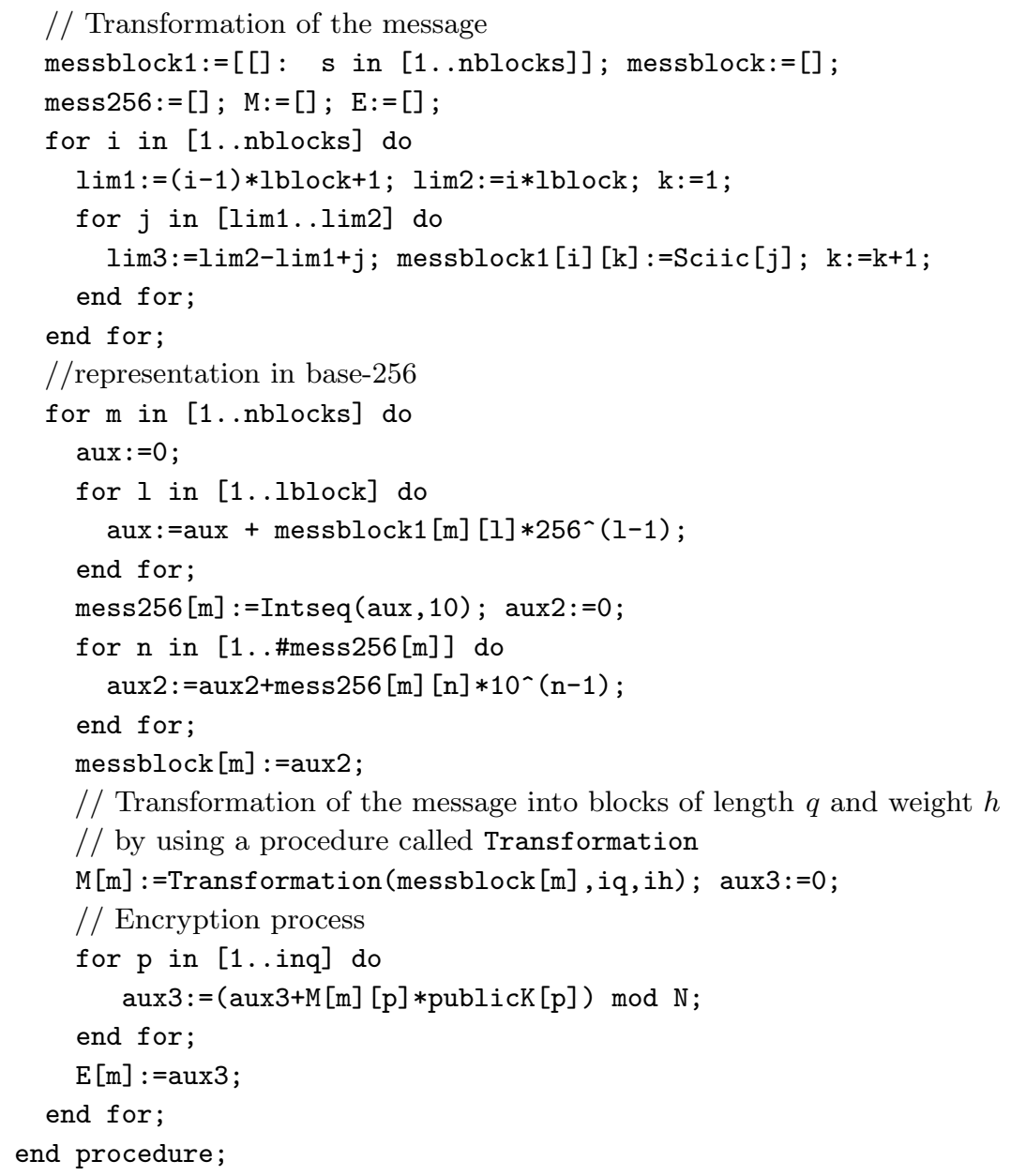

The procedure for decrypting an encrypted message, which was saved in a file, is the following.

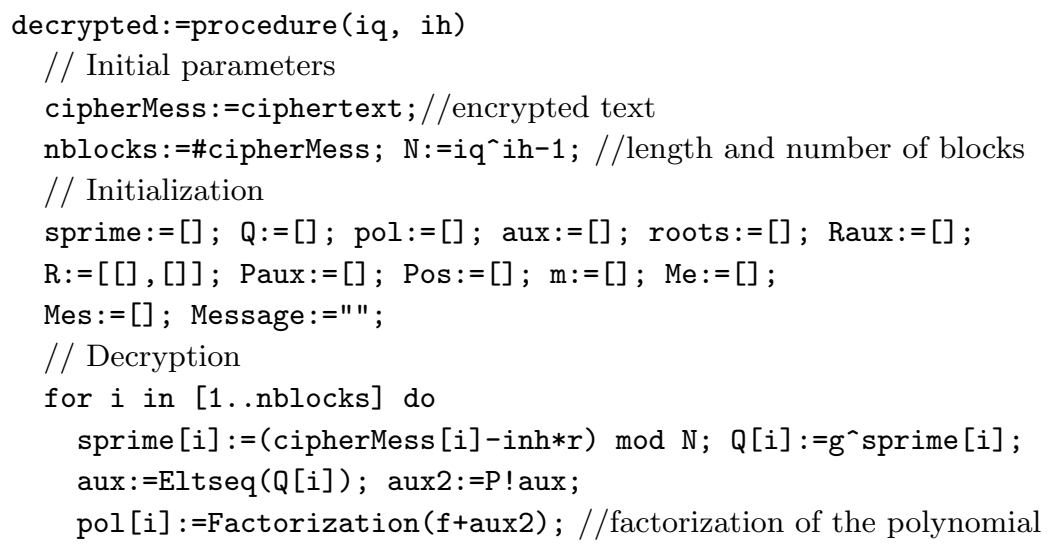




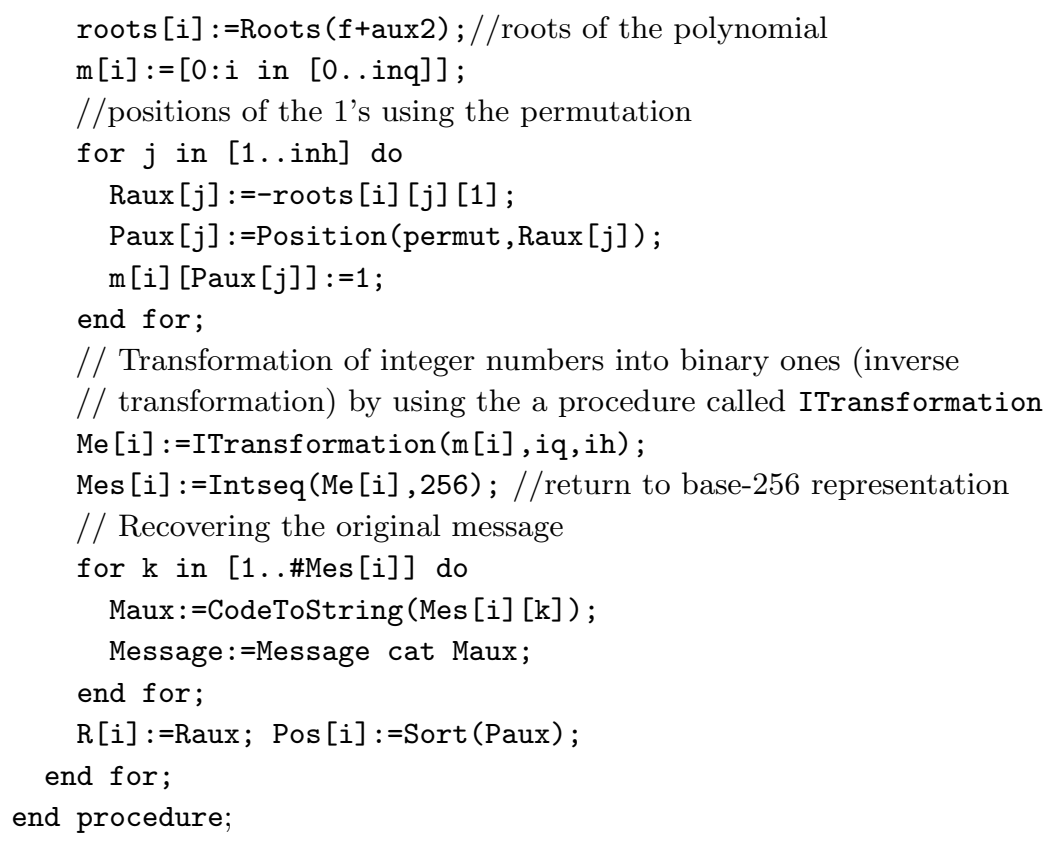

\section{Computational results}

We have encrypted and decrypted several messages with different lengths in order to measure the running time needed in different cases. To do this, we have considered the pairs of parameters listed in Table 2, and the results obtained are shown in Table 3.

For these pairs of values, we have considered Lenstra's conjecture ([11]). Thus, we have made public different number of elements of the public key. This number of published elements is represented by PKN and the length of each block of the message is given in parentheses. In this sense, we have measured the time to encrypt and decrypt messages considering a part of the whole public knapsack. For each value of $q$ we have considered a half part of them, then a quarter part, and finally the $10 \%$ of the public key.

The variables analyzed in this experimental computation are the following: Text length: TL (measured in KB); number of characters of the message: CM (adding 0's if needed); number of blocks of the message: BN; and the Encryption and Decryption Time (in seconds): ET and DT, respectively. The messages considered have $50 \mathrm{~KB}$ (around 49896 characters) and $192 \mathrm{~KB}$ (around 191727 characters).

Table 3: Experimental computational results for the pairs $(q, h)$ listed in Table 2.

\begin{tabular}{|c||c|c|c|c|c|c|}
\hline Parameters & PKN & TL & CM & BN & ET & DT \\
\hline \hline$(409,17)$ & 205 & 50 & 49900 & 4990 & 4.250 & 14.859
\end{tabular}


Table 3: Experimental computational results for the pairs $(q, h)$ listed in Table 2.

\begin{tabular}{|c||c|c|c|c|c|c|}
\hline Parameters & PKN & TL & CM & BN & ET & DT \\
\hline \hline & $(10)$ & 192 & 191730 & 19173 & 16.078 & 59.093 \\
\hline$(409,17)$ & 102 & 50 & 49896 & 7128 & 3.328 & 20.766 \\
& $(7)$ & 192 & 191730 & 27390 & 12.938 & 81.875 \\
\hline$(409,17)$ & 41 & 50 & 49892 & 12473 & 2.531 & 35.734 \\
& $(4)$ & 192 & 191728 & 47932 & 9.281 & 138.797 \\
\hline$(601,13)$ & 301 & 50 & 49896 & 5544 & 5.500 & 11.750 \\
& $(9)$ & 192 & 191727 & 21303 & 21.172 & 46.922 \\
\hline$(601,13)$ & 150 & 50 & 49896 & 7128 & 3.906 & 14.672 \\
& $(7)$ & 192 & 191730 & 27390 & 14.718 & 58.218 \\
\hline$(601,13)$ & 60 & 50 & 49895 & 9979 & 2.406 & 20.125 \\
& $(5)$ & 192 & 191730 & 38346 & 8.859 & 79.234 \\
\hline$(631,13)$ & 316 & 50 & 49896 & 5544 & 5.922 & 12.234 \\
& $(9)$ & 192 & 191727 & 21303 & 22.437 & 48.953 \\
\hline$(631,13)$ & 158 & 50 & 49896 & 7128 & 4.094 & 15.328 \\
& $(7)$ & 192 & 191730 & 27390 & 15.593 & 60.859 \\
\hline$(631,13)$ & 63 & 50 & 49895 & 9979 & 2.484 & 21.172 \\
& $(5)$ & 192 & 191730 & 38346 & 9.312 & 83.078 \\
\hline$(839,13)$ & 420 & 50 & 49900 & 4990 & 6.610 & 11.437 \\
& $(10)$ & 192 & 191730 & 19173 & 25.390 & 45.640 \\
\hline$(839,13)$ & 210 & 50 & 49896 & 6237 & 4.515 & 13.781 \\
& $(8)$ & 192 & 191728 & 23966 & 17156 & 54.875 \\
\hline$(839,13)$ & 84 & 50 & 49896 & 8316 & 2.562 & 18.016 \\
& $(6)$ & 192 & 191730 & 31955 & 9.672 & 70.781 \\
\hline$(599,13)$ & 300 & 50 & 49896 & 5544 & 5.562 & 11.953 \\
& $(9)$ & 192 & 191727 & 21303 & 21.047 & 47.750 \\
\hline$(599,13)$ & 150 & 50 & 49896 & 7128 & 3.921 & 14.953 \\
& $(7)$ & 192 & 191730 & 27390 & 14.625 & 56.250 \\
\hline$(599,13)$ & 60 & 50 & 49895 & 9979 & 2.360 & 20.485 \\
& $(5)$ & 192 & 191730 & 38346 & 8.875 & 80.500 \\
\hline$(797,13)$ & 399 & 50 & 49896 & 5544 & 8.093 & 12.562 \\
& $(9)$ & 192 & 191727 & 21303 & 31.046 & 49.750 \\
\hline$(797,13)$ & 199 & 50 & 49896 & 6237 & 4.218 & 13.640 \\
& $(8)$ & 192 & 191728 & 23966 & 16.094 & 54.234 \\
\hline$(597,13)$ & 80 & 50 & 49896 & 8316 & 2.500 & 17.844 \\
& $(6)$ & 192 & 191730 & 31955 & 9.187 & 70.094 \\
\hline & 286 & 50 & 49896 & 5544 & 5.140 & 11.656 \\
& $(9)$ & 192 & 191727 & 21303 & 19.594 & 46.468 \\
\hline & 143 & 50 & 49896 & 7128 & 3.625 & 14.594 \\
& $(7)$ & 192 & 191730 & 27390 & 13.844 & 57.796 \\
\hline & 57 & 50 & 49895 & 9979 & 2.328 & 19.875 \\
& & & & & & \\
& $15)$ & 192 & 191730 & 38346 & 8.453 & 78.266 \\
\hline
\end{tabular}


Table 3: Experimental computational results for the pairs $(q, h)$ listed in Table 2.

\begin{tabular}{|c||c|c|c|c|c|c|}
\hline Parameters & PKN & TL & CM & BN & ET & DT \\
\hline \hline$(577,13)$ & 289 & 50 & 49896 & 5544 & 5.249 & 11.172 \\
& $(9)$ & 192 & 191727 & 21303 & 19.797 & 44.860 \\
\hline$(577,13)$ & 144 & 50 & 49896 & 7128 & 3.704 & 14.016 \\
& $(7)$ & 192 & 191730 & 27390 & 13.968 & 55.578 \\
\hline$(577,13)$ & 58 & 50 & 49895 & 9979 & 2.266 & 19.203 \\
& $(5)$ & 192 & 191730 & 38346 & 8.703 & 75.234 \\
\hline$(167,17)$ & 84 & 50 & 49896 & 7128 & 2.703 & 15.625 \\
& $(7)$ & 192 & 191730 & 27390 & 10.156 & 62.016 \\
\hline$(167,17)$ & 42 & 50 & 49892 & 12473 & 2.500 & 26.609 \\
& $(4)$ & 192 & 191728 & 47932 & 9.359 & 104.281 \\
\hline$(167,17)$ & 20 & 50 & 49891 & 49891 & 4.453 & 104.312 \\
& $(1)$ & 192 & 191727 & 191727 & 16.438 & 406.844 \\
\hline
\end{tabular}

If all the pairs of the parameters listed in Table 3 are analyzed, the best pair for the encryption process is the last one, namely $(167,17)$. In this case, the block length is the smallest one. This value depends only on the values of $q$ and $h$ and is calculated with the expression $\log _{2}\left(\begin{array}{l}q \\ h\end{array}\right)$ (and divided by 8).

For the decryption process, the values are similar, and the best one is the pair $(577,13)$, with a block length equals to 10 bits.

Finally, as it could be observed, the decryption process is slower than the encryption process in all cases.

\section{Conclusions}

We have studied and implemented the Chor-Rivest cryptosystem with Magma software by using a larger range of safe parameters than those suggested in previous proposals. For these parameters no attack has been described. Moreover, we have presented a Magma implementation of this cryptosystem suitable for the set of these new parameters in an efficient way.

The implementation opens a door to the future in order to use realistically the Chor-Rivest cryptosystem, based on the fact that no attack has yet been proposed when the parameters $q, h$ determined above, are used.

Acknowledgement. Partially supported by CDTI, Ministerio de Industria, Turismo y Comercio (Spain) in collaboration with Telefónica I+D (Project SEGUR@) with reference CENIT-2007 2004.

\section{References}

[1] E.F. Brickell, Breaking iterated knapsacks, Lecture Notes in Comput. Sci. 1960 (1985) 342-358. 
[2] B. Chor, Two issues in public key cryptography. RSA bit security and a new knapsack type system, The MIT Press, Cambridge, MS, 1985.

[3] B. Chor, R.L. Rivest, A knapsack-type public key cryptosystem based on aritmethic in finite fields, IEEE Trans. Inform. Theory 34, 5 (1988) 901909 .

[4] M.J. Coster, A. Joux, B.A. LaMacchia, A.M. Odlyzko, C.P. Schnorr, J. Stern, Improved low-density subset sum algorithms, Comput. Complexity 2 (1992) 111-128.

[5] T.M. Cover, Enumerative source encoding, IEEE Trans. Inform. Theory 19 (1973) 73-77.

[6] L. Hernández Encinas, J. Muñoz Masqué, A. Queiruga Dios, Maple implementation of the Chor-Rivest cryptosystem, Lecture Notes in Comput. Sci. 3992 (2006) 438-445.

[7] L. Hernández Encinas, J. Muñoz Masqué, A. Queiruga Dios, Safer parameters for the Chor-Rivest cryptosystem, Comput. Math. Appl. 56 (2008) $2883-2886$.

[8] T. Izu, J. Kogure, T. Koshiba, T. Shimoyama, Low-density attack revisited, Des. Codes Crypt. 43 (2007) 47-59.

[9] A. Joux, R. Lercier, Improvements on the general number field sieve for discrete logarithms in prime fields. A comparison with the Gaussian integer method, Math. Comput. 72 (2003) 953-967.

[10] A. Joux, R. Lercier, N. Smart, F. Vercauteren, The number field sieve in the medium case, Lecture Notes in Comput. Sci. 4117 (2006) 326-344.

[11] H.W. Lenstra, Jr., On the Chor-Rivest knapsack cryptosystem, J. Cryptology 3 (1991) 149-155.

[12] A. Menezes, P. van Oorschot, S. Vanstone, Handbook of applied cryptography, CRC Press, Boca Raton, FL, 1997.

[13] R.A. Mollin, An introduction to cryptography, Chapman \& Hall/CRC, Boca Raton, FL, 2001.

[14] J.C. Lagarias, A.M. Odlyzko, Solving low-density subset-sum problems, J. ACM 32 (1985), 229-246.

[15] R.C. Pohlig, M.E. Hellman, An improved algorithm for computing logarithms over $G F(p)$ and its cryptographic significance, IEEE Trans. Inform. Theory 24 (1978) 106-110.

[16] O. Schirokauer, D. Weber, T. Denny, Discrete logarithms: the effectiveness of the index calculus method, Lecture Notes in Comput. Sci. 1122 (1996) $337-361$. 
[17] S. Vaudenay, Cryptanalysis of the Chor-Rivest cryptosystem, J. Cryptology 14 (2001) 87-100.

[18] B. Wang, Q. Wu, Y. Hu, A knapsack-based probabilistic encryption scheme, Inform. Sci. 177 (2007), 3981-3994.

[19] A.M. Youssef, Cryptanalysis of a knapsack-based probabilistic encryption scheme, Information Sciences 179 (2009) 3116-3121. 